\title{
Slab Delivery of Incoherent Pump Light to Double-Clad Fiber Amplifiers: Numerical Simulations
}

\author{
Patrick Kano, Dmitrii Kouznetsov, Jerome V. Moloney, and Moysey Brio
}

\begin{abstract}
Compact fiber amplifiers with tapered slab delivery of the pump are analyzed numerically. A procedure for the selection of the structural parameters of this device is presented together with a simple asymptotic estimate for the efficiency. Simulations based on the scalar paraxial beam propagation equation demonstrate the validity of this estimate.
\end{abstract}

Index Terms-Double-clad fiber, optical fiber amplifiers, scalar paraxial beam propagation equation.

\section{INTRODUCTION}

$\mathbf{E}$ FFICIENTLY pumping a fiber over a distance of a few centimeters is a technical problem of current interest. A tapered slab geometry, Fig. 1, has recently been proposed as a compact double-clad fiber amplifier design [1]-[5]. Asymptotic estimates for the absorption efficiency and the minimum slab length for reasonable values of the structural parameters have also been suggested [6]. These estimates, however, are based on strong assumptions and require numerical consideration. In this paper, we choose the structural parameters based on the criteria in [6], numerically simulate the device using the scalar beam propagation equation, investigate the accuracy of the estimate for the efficiency, and suggest a domain of validity.

\section{Structure PARAMETERS AND AsYMPtotic Estimates}

The selection of parameters which are both physically realistic and provide efficient pumping is clearly a crucial step in the device's construction. The following presents the choice of parameters for the structure numerically analyzed in this paper.

First to consider is the choice of fiber. The core and fiber radii and the indices of refraction of the core, fiber cladding, and surrounding coolant should allow for single mode propagation of the signal in the core. This requires that the normalized

Manuscript received February 19, 2004; revised May 6, 2004. This work was supported by the Air Force Office of Scientific Research under Grant F49620-02-1-0380.

P. Kano, J. V. Moloney, and M. Brio are with the Arizona Center for Mathematical Sciences, Department of Mathematics, University of Arizona, Tucson, AZ 85721 USA (e-mail: pkano@math.arizona.edu; jml@acms.arizona.edu; brio@math.arizona.edu).

D. Kouznetsov is with the Arizona Center for Mathematical Sciences, Department of Mathematics, University of Arizona, Tucson, AZ 85721 USA and also with the Institute for Laser Science, University of Electro-Communications, Tokyo, 182-8585, Japan (e-mail: dima@ acms.arizona.edu; dima@ils.uec.ac.jp).

Digital Object Identifier 10.1109/JQE.2004.833232

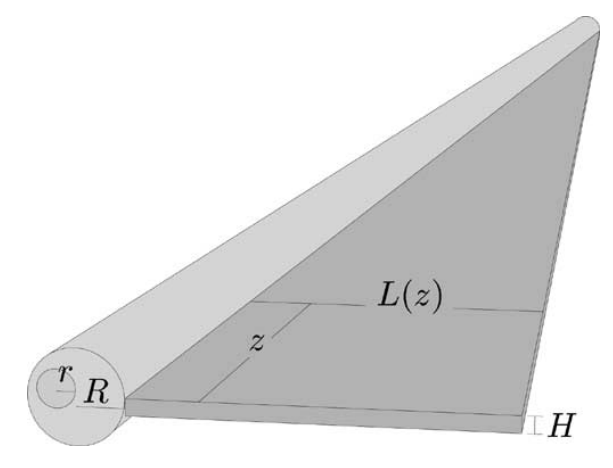

Fig. 1. Tapered slab pumped double-clad fiber.

TABLE I

FIBER PARAMETERS

\begin{tabular}{c|c|c|c|c}
\hline$r_{\text {core }}$ & $R_{\text {fiber }}$ & $n_{\text {coolant }}$ & $n_{\text {cladding }}$ & $n_{\text {core }}$ \\
\hline $10 \mu \mathrm{m}$ & $30 \mu \mathrm{m}$ & 1.330 & 1.560 & 1.561 \\
\hline
\end{tabular}

frequency of the fiber be less than 2.4 [7]. We choose the values summarized in Table I.

The index of refraction of the coolant corresponds to a value near that for the organic liquid coolants often used for double clad devices. The core and cladding have indices typical of silica glasses. The imaginary part of the core index of refraction, which is related to the absorption, remains a parameter. To select a wavelength for the signal, we consider an $\mathrm{Er} / \mathrm{Yb}$ core [8]. This material emits predominantly at $1.535 \mu \mathrm{m}$. The center of the core is offset from the center of the fiber, $9 \mu \mathrm{m}$ up and $1 \mu \mathrm{m}$ left. For these parameters, $V \approx 2.287$.

Next, one must choose a source and slab. Since erbium/ ytterbium codopants in a phosphate glass have a maximum absorption $\alpha \approx 10 \mathrm{~cm}^{-1}$ near $\lambda=0.98 \mu \mathrm{m}$, we select a source with this wavelength. If one assumes to have a perfectly matched single mode slab pump in the $y$-direction, then the number of modes generated by the pump is approximately given by the formula

$$
N_{\text {Pump }}=2 \frac{(\mathrm{NA}) L_{0}}{\lambda}
$$

where (NA) is the numerical aperture of the pump in the $\mathrm{x}$-direction [6]. For a slab of initial width $L_{0}=100 \mu \mathrm{m}$, thickness $H=6 \mu \mathrm{m}$, and an NA $=0.15$, the number of modes created by this pump is $N_{\text {Pump }} \approx 30$.

One must also compute the slab index of refraction required so that the lowest order modes of the fiber have strong overlap 
TABLE II

SLAB AND PUMP PARAMETERS

\begin{tabular}{c|c|c|c|c}
\hline$L_{0}$ & $H$ & $n_{\text {slab }}$ & $N_{\text {Pump }}$ & $\lambda$ \\
\hline $100 \mu \mathrm{m}$ & $6 \mu \mathrm{m}$ & 1.567 & 30 & $0.98 \mu \mathrm{m}$ \\
\hline
\end{tabular}

TABLE III

SLAB LENGTHS AND $\Lambda$ FOR VARIOUS ABSORPTION COEFFICIENTS

\begin{tabular}{c|c|c|c}
\hline$\alpha\left(\mathrm{cm}^{-1}\right)$ & $T_{0}(\mathrm{~cm})$ & $\Lambda_{\text {clad core }}(\mathrm{cm})$ & $\Lambda_{\text {unclad core }}(\mathrm{cm})$ \\
\hline 1 & $6 / \pi \approx 1.9099$ & 10.9099 & 2.9099 \\
2 & $3 / \pi \approx 0.9549$ & 5.4549 & 1.4549 \\
4 & $3 /(2 \pi) \approx 0.4775$ & 2.7275 & 0.7275 \\
8 & $3 /(4 \pi) \approx 0.2387$ & 1.3637 & 0.3637 \\
16 & $3 /(8 \pi) \approx 0.1194$ & 0.6819 & 0.1819 \\
\hline
\end{tabular}

with the highest order modes of the slab at $z=0$. In [6], this condition has been shown to reduce to the statement

$$
n_{\text {slab }}=\frac{n_{\text {cladding }}}{\left(\frac{\text { cos NA }}{n \text { cladding }}\right)} .
$$

For the present case, $n_{\text {slab }} \approx 1.567$. Table II provides a summary of the slab and pump parameters.

The final step is to determine the tapered slab length $T$ in the estimate of the absorption efficiency for $z<T$ [6]

$$
\begin{aligned}
\eta & =1-\frac{\|E(z)\|^{2}}{\|E(0)\|^{2}} \\
& =1-\left(1-\frac{z}{\Lambda}\right)^{\frac{\alpha \pi r^{2}}{H L_{0}} T}
\end{aligned}
$$

where

$$
\Lambda=T \frac{\pi R^{2}+H L_{0}}{H L_{0}} .
$$

The absorption rate of the core $\alpha$ is given by the equation $(d / d z)\|E\|^{2}=-\alpha\|E\|^{2}$, where $\|E\|^{2}=\int_{\Omega}|E|^{2} d x d y$. The parameter $\Lambda$ can be interpreted as an effective absorption length.

In order to allow for a uniform intensity of the pump in the fiber, the length of the slab is chosen so that the exponent in the efficiency is one [6]

$$
T_{0}=\frac{H L_{0}}{\alpha \pi r^{2}}
$$

Finally, if one defines

$$
\alpha_{\text {effective }} \equiv \alpha \frac{\pi r^{2}}{\pi R^{2}+H L_{0}}
$$

the the expressions for $\Lambda$ and $\eta$ can be written concisely

$$
\begin{aligned}
\Lambda\left(T_{0}\right) & =\frac{1}{\alpha_{\text {effective }}} \\
\eta(z) & =z \alpha_{\text {effective }}
\end{aligned}
$$

The efficiency is thus predicted to be directly proportional to the propagation distance. Obviously, the fiber can be extended longer than $T_{0}$ in order to use the part of the pump which is not absorbed but still guided. In Table III are the tapering length and approximate $\Lambda\left(T_{0}\right)$ for physically relevant values of $\alpha$ and the parameters given above.

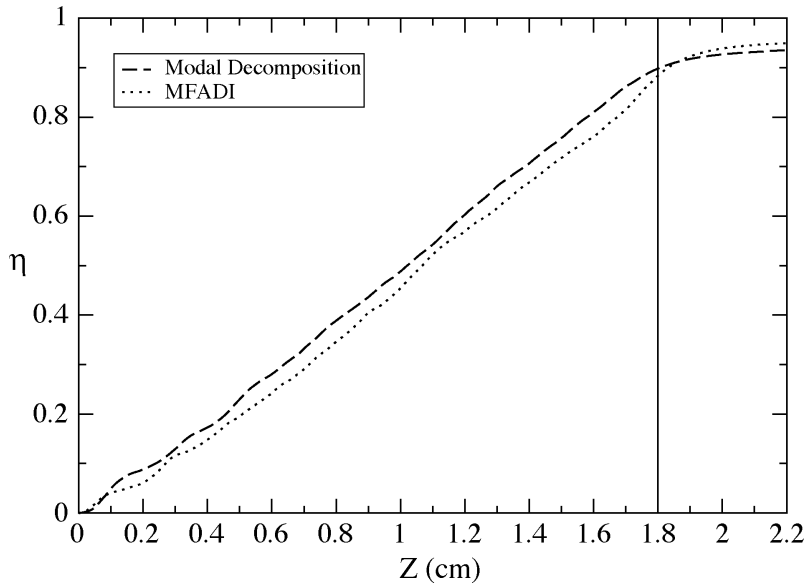

Fig. 2. Efficiency $\eta$ versus propagation distance measured from simulations using the MFADI (dotted line) and modal decomposition (dashed line) codes.

\section{NUMERICAL COMPUTATIONS AND DISCUSSION}

\section{A. Scalar Paraxial Beam Propagation Method}

The scalar paraxial beam propagation equation

$$
\frac{\partial E}{\partial z}=\frac{1}{2 i \beta}\left[\nabla_{\perp}^{2}-\left(\beta^{2}-k_{0}^{2} n^{2}\right)\right] E
$$

where $\beta$ is the propagation constant, $k_{0}$ is the free space wave number, $n=n(x, y, z)$ is a complex index of refraction, and $E$ is the envelope to one transverse component of the electric field, and is a standard tool for the numerical simulation of photonic structures [9]-[11]. Here, this equation is solved by the split-step Mitchell-Fairweather alternating direction implicit (MFADI) method [12], [13]. Perfectly reflecting boundary conditions are implemented to conserve the total energy in the computational domain. The propagation constant $\beta$ is adaptively determined by [14]

$$
\beta^{2}=\frac{\int_{\Omega}\left|k_{0} n E\right|^{2}-\left|\nabla_{\perp} E\right|^{2} d x d y}{\int_{\Omega}|E|^{2} d x d y} .
$$

One test of the code has been to simulate the tapered slab coupled to an unclad core studied in [6]. The indices of refraction are $n_{\text {slab }}=1.58, n_{\text {core }}=1.56, n_{\text {coolant }}=1.54$. The core has a $4.3-\mu \mathrm{m}$ radius and a local absorption coefficient of $10 \mathrm{~cm}^{-1}$. The slab has a $6-\mu \mathrm{m}$ thickness, $1-\mathrm{cm}$ initial width, and a length of $1.8 \mathrm{~cm}$.

The initial condition utilized to model the diode pump consists of an incoherent combination of slab modes in the $x$-direction with a single mode in the $y$-direction [5]. In particular, $E(x, y)=\mathcal{X}(x) \mathcal{Y}(y)$, where

$$
\begin{aligned}
& \mathcal{X}(x)= \begin{cases}\sum_{m} \sin \left(\frac{\pi m}{L_{0}}\left(x+\frac{L_{0}}{2}\right)\right) e^{\left(i \phi_{m}\right)}, & -\frac{L_{0}}{2} \leq x \leq \frac{L_{0}}{2} \\
0, & \text { else }\end{cases} \\
& \mathcal{Y}(y)= \begin{cases}\cos \left(\kappa \frac{H}{2}\right) e^{\sigma\left(\frac{H}{2}+y\right),} & y<-\frac{H}{2} \\
\cos (\kappa y), & -\frac{H}{2} \leq y \leq \frac{H}{2} \\
\cos \left(\kappa \frac{H}{2}\right) e^{\sigma\left(\frac{H}{2}-y\right)}, & y>\frac{H}{2}\end{cases}
\end{aligned}
$$



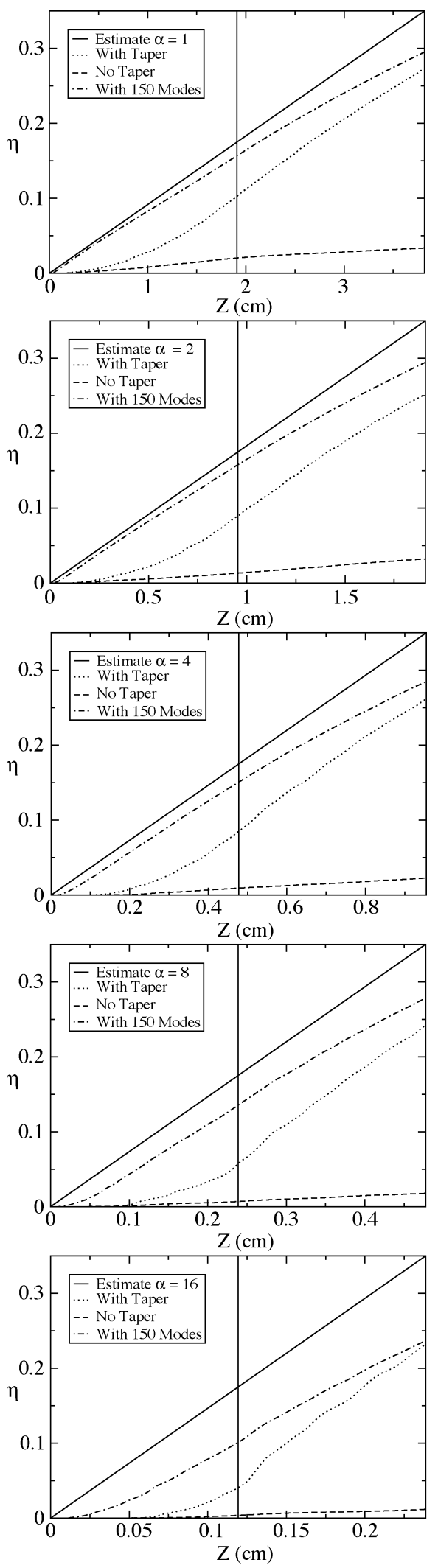

Fig. 3. Absorption efficiency $\eta$ for $\alpha=1,2,4,8,16 \mathrm{~cm}^{-1}$. The estimated efficiency using (8) is indicated by a solid curve.

where $x, y$ refer to the coordinate system with its origin at the center of the lateral cross section of the slab. The phase $\phi_{m} \in[-\pi, \pi]$ for each mode ensures that the initial condition

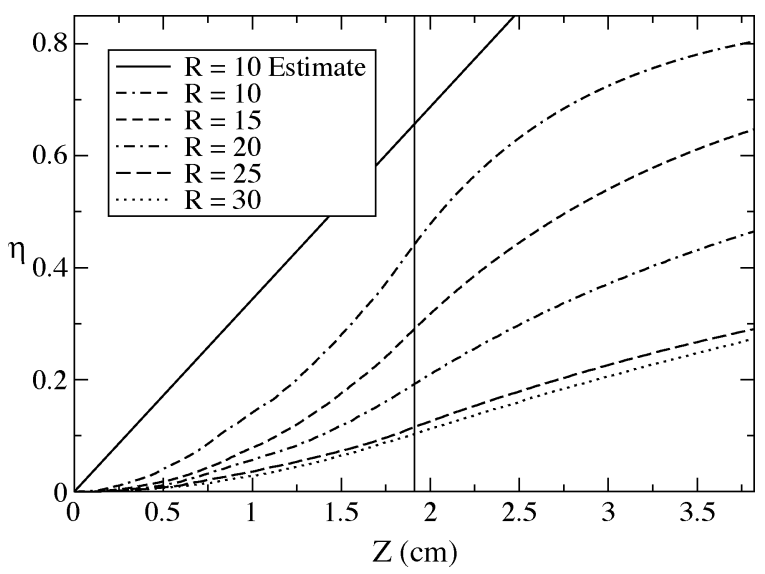

Fig. 4. Absorption efficiency $\eta$ versus the fiber radius $R$ for $\alpha=1$.

is random as it would be from a diode pump. The coefficients $\kappa$ and $\sigma$ solve the system of equations

$$
\begin{aligned}
\kappa^{2}+\sigma^{2} & =k^{2}\left(n_{\text {slab }}^{2}-n_{\text {coolant }}^{2}\right) \\
\tan \left(\kappa \frac{H}{2}\right) & =\frac{\sigma}{\kappa} .
\end{aligned}
$$

Finally, to emulate the effect of tilting the light toward the fiber, the initial field is multiplied by the phase factor $e^{i p_{0}\left(x+\left(L_{0} / 2\right)\right)}=$ $e^{i\left(0.2(\mu \mathrm{m})^{-1}\left(x+\left(L_{0} / 2\right)\right)\right)}$. This corresponds to a horizontal velocity $v_{x}=\left(-p_{0} / \beta\right)$ and an inclination of the pump with respect to the core of approximately $1.3^{\circ}$. Fig. 2 shows the evolution of the efficiency averaged over many realizations of the initial conditions. The vertical line indicates the length of the tapered slab. The agreement between the previous computations [6] based on a modal decomposition and those from the MFADI method is clear.

\section{B. Comparison and Results}

In Fig. 3 are results from simulating the structure with the parameters in Section II and the initial conditions in III-A. The structure with a tapered slab is clearly more efficient than the one with a slab which is not tapered. As anticipated, the rate of absorption is approximately linear and has a slope near that of $\alpha_{\text {effective }}$. The estimate is, however, considerably more than that measured, especially for larger values of $\alpha$. The discrepancy in the absorption can be attributed to two sources. One is the thickness of the cladding. With cladding, the pump light requires additional distance to diffuse through the fiber before it can be absorbed in the core. As seen in Fig. 4, reducing the thickness of the cladding improves the absorption efficiency. An additional cause for the delay is the lack of sufficient high order modes in the initial condition. The estimate proposed in [6] is predicated on the assumption that the slab and fiber modes have strong overlap and that the highest order modes of the slab are quickly expelled as the width of the slab is decreased. If the initial condition contains an insufficient number of higher order modes then there occurs a delay in the transfer of light from the tapered slab to the fiber until the resonance condition is fulfilled. Simulating an initial condition where the number of modes is increased beyond the suggested number of $N_{\text {Pump }}=\left((\mathrm{NA}) L_{0} k_{0} / \pi\right) \approx 30$ modes delivers the improvement seen in Fig. 5. From the simulations in (3) it 


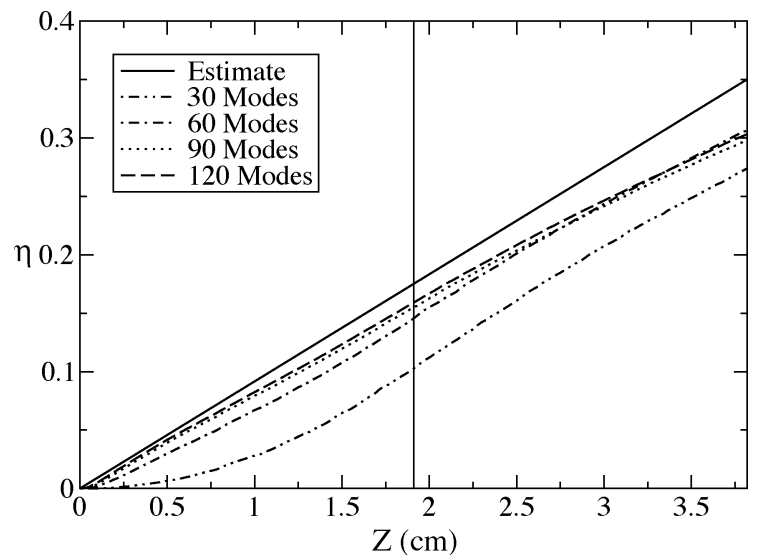

Fig. 5. Absorption efficiency $\eta$ versus initial condition modes for $\alpha=1$.

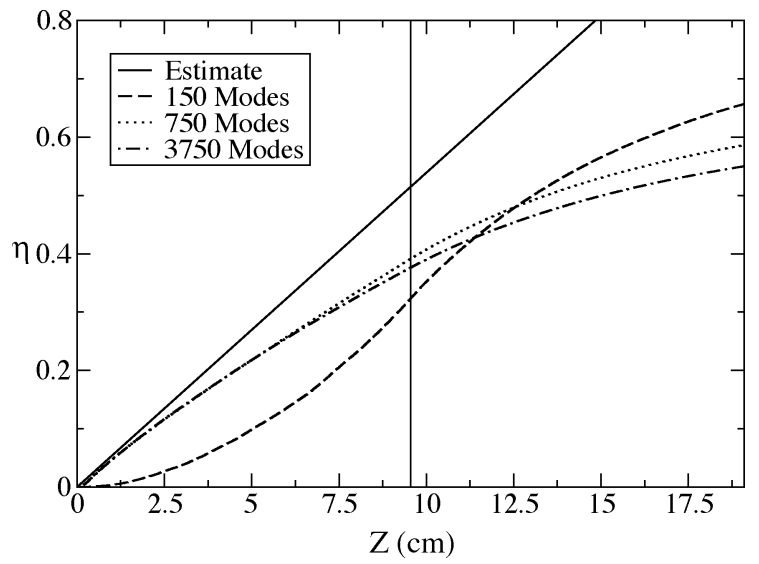

Fig. 6. Absorption efficiency $\eta$ for slab width $500 \mu \mathrm{m}$ and $\alpha=1$.

is also clear that increasing the number of modes to 150 has the best effect for small values of $\alpha$. Finally, in Fig. 6 we see that the behavior is reproduced in a wider slab with a $500-\mu \mathrm{m}$ width. For this case, $T_{0} \approx 9.5493 \mathrm{~cm}, \Lambda_{0} \approx 18.5493 \mathrm{~cm}$ and all other parameters are identical to the $100-\mu \mathrm{m}$ width slab. Again, the efficiency is approximately linearly increasing and shows better agreement with the estimate for a more random initial condition.

\section{CONCLUSIONS}

The tapered slab geometry is promising as a compact and efficient pump design. The simulations show that the linear efficiency estimate suggested in [6] is accurate over a few centimeters for small values of $\alpha<5 \mathrm{~cm}^{-1}$ and highly random initial conditions. The delay in the absorption observed for initial conditions with few modes can be overcome by adding higher order modes. Greater efficiency can also be achieved by reducing the thickness of the fiber cladding. The simplicity of the expression for the efficiency makes it an attractive tool for designing tapered slab pumps. An experimental investigation of the tapered slab geometry is suggested as a source for future work.

\section{ACKNOWLEDGMENT}

The authors would like to thank M. Kolesik, A. Mafi, G. Katona, M. Mansuripur, V. Temyanko, and Nasser
Peyghambarian at the Arizona Center for Mathematical Sciences and the Optical Science Center, University of Arizona, Tuscon, for stimulating discussions.

\section{REFERENCES}

[1] D. Kouznetsov, J. V. Moloney, and E. M. Wright, "Efficiency of pump absorption in double-clad fiber amplifiers. 1. Fiber with circular symmetry," J. Opt. Soc. Amer. B, vol. 18, no. 6, pp. 743-749, 2001.

[2] D. Kouznetsov and J. V. Moloney, "Efficiency of pump absorption in double-clad fiber amplifiers. 2. Broken circular symmetry," J. Opt. Soc. Amer. B, vol. 19, no. 6, pp. 1259-1263, 2002.

[3] — , "Efficiency of pump absorption in double-clad fiber amplifiers. 3. Calculation of modes," J. Opt. Soc. Amer. B, vol. 19, no. 6, pp. 1304-1309, 2002.

[4] D. Kouznetsov and J. Moloney, "Highly efficient, high-gain, short wavelength and power scalable incoherent diode slab-pumped fiber laser amplifier," in IEEE J. Quantum Electron., vol. 39, Nov. 2003, pp. $1452-1461$, to be published.

[5] —-, "Highly efficient, high-gain, short length and power scalable incoherent diode slab-pumped fiber amplifier/laser," IEEE J. Quantum Electron., vol. 39, pp. 1452-1461, Nov. 2003.

[6] - "Slab delivery of incoherent pump light to double-clad fiber amplifiers: an analytic approach," IEEE J. Quantum Electron., to be published.

[7] C. Yeh, Handbook of Fiber Optics: Theory and Applications. New York: Academic, 1990

[8] G. C. Valley, "Modeling cladding-pumped Er/Yb fiber amplifiers," Opt. Fib. Technol., vol. 7, pp. 21-44, 2001.

[9] G. Lifante, Integrated Photonics: Fundamentals. New York: Wiley, 2003.

[10] K. Okamoto, Fundamentals of Optical Waveguides. New York: Academic, 2000.

[11] Y. Tsuji and M. Koshiba, "Finite element beam propagation method with perfectly matching layer boundary conditions for three-dimensional optical waveguides," Int. J. Numer. Model., vol. 13, pp. 115-116, 2000.

[12] J. Strikwerda, Finite Difference Schemes and Partial Differential Equations. Belmont, CA: Wadsworth, 1989.

[13] P. Kano, Ph.D. dissertation, University of Arizona, Tuscon, to be published.

[14] F. Schmidt, "An adaptive approach to the numerical simulation of Fresnel's wave equation," J. Lightwave Technol., vol. 11, pp. 1425-1434, Sept. 1993.

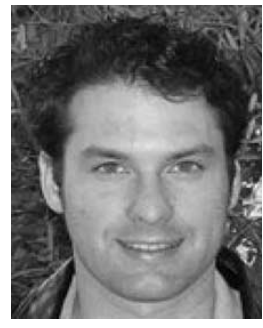

Patrick Kano received the B.S. degree from the University of Nevada, Reno, in 1998, and the Diplom-Physik degree from Dresden University of Technology, Germany, in 2000.

His research interests computational photonics, numerical inversion of the Laplace transform, and pattern formation.

Mr. Kano is a member of the Society for Industrial and Applied Mathematics.

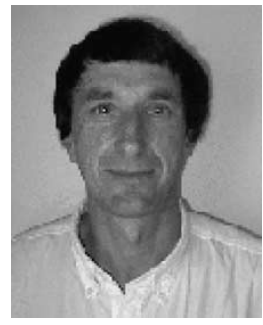

Dmitrii Kouznetsov received the B.Sc. degree from Moscow State University, U.S.S.R., in 1980, and the Ph.D. degree from Lebedev Physics Institute, Moscow, in 1988.

He was with Lebedev Physics Institute in 1980, the Mexican National University, Mexico City, in 1992, and the Arizona Center for Mathematical Sciences, Tucson, in 2000. His research interests have included new methods of active image formation, proof of quantum stability of optical soliton, theorems on quantum noise of nonlinear amplifiers, methods of random wavevectors in simulation of correlated processes with wide spectra, calculation of efficiency of resonant backscattering of sound by sound, the concept of continuum of modes in quantum optics, theorems on boundary behavior of modes of Dirichlet Laplacian, the application to double-clad fibers, and the concept of slab delivery of pump to fiber amplifiers. Dr. Kouznetsov is a member of Optical Society of America. 


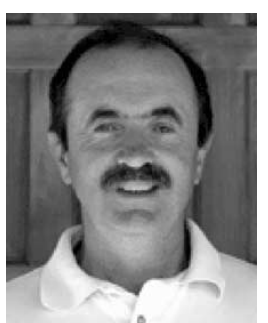

Jerome V. Moloney received the B.Sc. degree from the University College, Cork, Ireland, in 1970, and the Ph.D. degree from the University of Western Ontario, ON, Canada, 1977.

$\mathrm{He}$ is currently a Director, Arizona Center for Mathematical Sciences Professor of Mathematics, University of Arizona, Tuscon, a Professor of Optical Sciences, and a Visiting Professor of Computational Physics, University College Cork, Ireland. He was with the Optical Sciences Center as a Research Associate from 1979 to 1981, a Research Assistan Professor from 1981 to 1984, and a Research Associate Professor from 1984 to 1985. He joined the Department of Mathematics and Optical Sciences Center, University of Arizona, in 1985. He was also with the Heriot-Watt University, Edinburgh, Scotland as a Reader in Physics from 1984 to 1990 and the Universität Bielefeld, Germany: Fakultät für Physik, as a Research Associate (Wissenschaftlicher Mitarbeiter), from 1977 to 1979. His research interests include mathematical modeling and simulation of photonics systems including semiconductor lasers, fiber lasers, photonic Bragg and photonic crystal fibers, fundamental theory of semiconductor lasers including microscopic physics, modeling high-power femtosecond atmospheric light string, nonlinear theory of partial differential equations and chaos synchronization in extended complex spatiotemporal interacting systems, sophisticated algorithm development for large scale computational photonics systems simulations including adaptive mesh refinement, and parallelization on distributed and shared memory supercomputer platforms.

Dr. Maloney is a member of the National Research Council, Naval Studies Board, Workshop on Mathematics, Mathematics in Material Sciences, Society for Industrial and Applied Mathematics. He is an organizer for the Institute of Mathematics and Its Applications Committee, University of Minnesota, Minneapolis, Workshop on Nonlinear Optical Materials

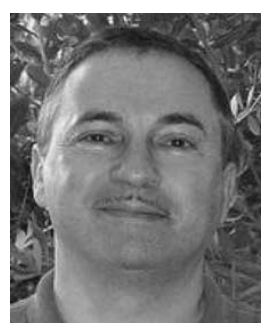

Moysey Brio received the B.A. degree from Latvian State University, Riga, in 1977, and the M.S. and Ph.D. degrees from University of California, Los Angeles, (UCLA) in 1981 and 1984, respectively.

$\mathrm{He}$ was a Visiting Scientist, Plasma Physics Group, UCLA, from 1984 to 1986, an Assistant Professor from 1987 to 1993, and an Associate Professor from 1993 to the present. He was a Visiting Scientist, Instituto Nacional de Mathemática Pure e Aplicada (IMPA) from 1994 to 1995, and a Visiting Scientist, Courant Institute of Mathematics, UCLA, from 1986 to 1987. His research includes design of high-resolution numerical methods for treatment of singularities with applications to plasma physics, nonlinear optics, and photonics. 